COMMENT. This study confirms the high morbidity in survivors of TBI in infants, and sequelae may be manifested only after long-term follow-up. The authors emphasize the importance of environmental factors in behavior and development of these patients.

Executive functions after traumatic brain injury in children are reviewed at Baylor College of Medicine, Houston, TX (Levin HS, Hanten G. Pediatr Neurol August 2005;33:79-93). Executive functions include cognitive control (decision making, planning, achieving goals), self-regulation, motivation, and conforming to social behavior. Inhibition is an age-dependent skill linked to cognitive control, and impaired in TBI as well as ADHD. The executive dysfunction following TBI resembles that seen with "developmental" ADHD, and both are related to frontal lobe involvement, which in TBI may be progressive.

\title{
SKULL X-RAYS FOR HEAD INJURY
}

The effect of abolishing skull X-rays on the rate of admission, use of computer tomography (CT), radiation dose per head injury, and detection of intracranial injuries was determined in patients, aged 1 to 14 years, presenting to the ED at Royal Hospital for Sick Children, Edinburgh, UK. 1535 patients with head injury seen between Aug 1, 1998 and July 31, 1999 (control period) were compared to 1867 presenting between Aug 1, 2002 and July 31,2003 (first year of new skull X ray policy). By abolishing skull X rays, about 400 normal $\mathrm{x}$ rays were avoided, CT orders rose from $1.0 \%$ to $2.1 \%$, with an unchanged positive $\mathrm{CT}$ rate $(25.6 \%$ v $25.0 \%)$, and no change in identification of intracranial injury $(0.2 \%$ v $0.4 \%)$ or in rates of neurosurgical intervention ( $0 \% \vee 0.1 \%)$. The admission rate was unchanged $(10.9 \% \mathrm{v}$ $10.1 \%$ ), and the radiation dose per head injury was slightly decreased from 0.045 to 0.042 $\mathrm{mSv}$. History of the injury and Glasgow coma scale are the best indicators of significant head injury in children. (Reed MJ, Browning JG, Wilkinson AG, Beattie T. Can we abolish skull x rays for head injury? Arch Dis Child August 2005;90:859-864). (Respond: Dr Matthew J Reed, Accident and Emergency Department, Royal Hospital for Sick Children, Sciennes Rd, Edinburgh, UK).

COMMENT. The abolition of skull X-rays in children aged 1 to 14 , presenting with head injury in an ED in the UK, does not increase the admission rate, individual radiation dose, or missed intracranial injury. The mechanism of head injury (falls of $>1$ meter, and road traffic accidents), drowsiness or loss of consciousness, and a low score on the Glasgow coma scale are the most reliable indicators of serious head injury and need for investigation and admission. As pointed out by Tasker RC, in a Commentary (Arch Dis Child 2005;90:774-775), the policy of abolishing skull X-rays doubled the rate of exposure to CT with overall increased risk of radiation. The debate regarding the utility of skull X-rays or the increased use of CT scans in EDs will continue. The above study and that of other guidelines (Dunning J et al. Arch Dis Child 2004;89:763-767) should help in the development of optimal head injury protocols for children. 